\title{
Diatomáceas epilíticas de águas oligotróficas e ácidas do Sudeste do Brasil ${ }^{1}$
}

\author{
Luís Gustavo de Castro Canani ${ }^{2,5}$, Mariângela Menezes ${ }^{3}$ e Lezilda Carvalho Torgan ${ }^{4}$
}

Recebido em 3/04/2010. Aceito em 10/01/2011

\section{RESUMO}

(Diatomáceas epilíticas de águas oligotróficas e ácidas do Sudeste do Brasil). Poucos estudos tem abordado a flora de diatomáceas de sistemas oligotróficos do Brasil. São apresentados os resultados da composição taxonômica e distribuição espacial e temporal de diatomáceas no rio do Salto, situado no Parque Estadual do Ibitipoca, Minas Gerais $\left(21^{\circ} 40^{\prime}-21^{\circ} 44^{\prime} \mathrm{S}\right.$ e $\left.43^{\circ} 52^{\prime}-43^{\circ} 55^{\prime} \mathrm{W}\right)$. As águas desse rio possuem $\mathrm{pH}$ entre 4,2 e 5,5 e concentração de ortofosfato entre 1,37 e 10,98 $\mu \mathrm{g} . \mathrm{L}^{-1}$. O estudo baseia-se na análise de 80 amostras coletadas em oito estações no leito rochoso do rio, em janeiro e julho de 2004, períodos de chuva e estiagem, respectivamente. Um total de 15 espécies e duas variedades taxonômicas, distribuídas em oito gêneros e oito famílias foram descritas e ilustradas, com destaque para o gênero Eunotia Ehrenberg. O estudo sobre diatomáceas bentônicas em ambientes lóticos é pioneiro no estado de Minas Gerais, sendo registradas 14 novas citações, das quais Fragilariforma stevensonii Metzeltin \& Lange-Bertalot, Eunotia pirarucu Metzeltin \& Lange Betalot, Nupela giluwensis Vyverman \& Compère e Sellaphora blackfordensis Mann \& Droop são primeiros registros para o Brasil.

Palavras-chave: Bacillariophyta, taxonomia, ambiente lótico

\begin{abstract}
(Epilithic diatoms from acidic and oligotrophic waters in Southeast Brazil) Few studies have been done on the diatom flora of acidic and oligotrophic environments from Brazil. We present the taxonomic composition and distribution of diatoms of Salto River, situated at Ibitipoca State Park, Minas Gerais $\left(21^{\circ} 40^{\prime}-21^{\circ} 44^{\prime} \mathrm{S} ; 43^{\circ} 52^{\prime}-43^{\circ} 55^{\prime} \mathrm{W}\right)$. The pH values ranged from 4.2 to 5.5 and orthophosphate was between 1.37 and $10.98 \mu \mathrm{g} . \mathrm{L}^{-1}$. The study is based on the analysis of 80 samples collected at eight stations in the rocky stream bed, in January and July 2004, rainy and dry seasons, respectively. A total of 15 species and two taxonomic varieties distributed in eight genera and eight families were described and illustrated, notably the Eunotia Ehrenberg genus. The study of benthic diatoms in lotic systems is pioneer in Minas Gerais state; 14 new records were recorded of which Fragilariforma stevensonii Metzeltin \& Lange-Bertalot, Eunotia pirarucu Metzeltin \& Lange-Bertalot, Nupela giluwensis Vyverman \& Compère and Sellaphora blackfordensis Mann \& Droop are first records for Brazil.
\end{abstract}

Key words: Bacillariophyta, taxonomy, lotic system

\section{Introdução}

As diatomáceas bentônicas são importantes produtores primários em ambientes lóticos e vem sendo utilizadas para avaliação e monitoramento da qualidade de água (Stevenson 1996). A quase totalidade dos estudos tem focado espécies indicadoras de eutrofia, já que grande parte dos ambientes se tornou ou está prestes a se tornar eutrófico, priorizando rios que recebem impacto da atividade humana. Pouca atenção vem sendo dada à flora de diatomáceas de ambientes oligotróficos (Kociolek \& Stoermer 2009). Entre os raros estudos realizados merece destaque o de Lange-Bertalot \& Metzeltin (1996), que em apenas três lagos oligotróficos na Europa identificaram 800 taxa e descreveram mais de 100 como novos ou que não puderam ser identificados. Uma contribuição adicional foi a criação da "lista vermelha" de espécies de diatomáceas que são raras e indicadoras de condições de pureza (oligotrofia) da água cujos habitats merecem especial atenção em termos de conservação (Lange-Bertalot 1996).

\footnotetext{
Parte da dissertação de Mestrado do primeiro Autor

2 Universidade Federal do Rio Grande do Sul, Programa de Pós-Graduação em Botânica, Porto Alegre, RS, Brasil

3 Universidade Federal do Rio de Janeiro, Museu Nacional, Rio de Janeiro, RJ, Brasil

4 Fundação Zoobotânica do Rio Grande do Sul, Museu de Ciências Naturais, Porto Alegre, RS, Brasil

5 Autor para correspondência: lgustavocc@gmail.com
} 
O conhecimento sobre a flora de diatomáceas bentônicas em águas oligotróficas e ácidas no Brasil é escasso e limita-se à contribuição de Schneck et. al. (2008). Outros estudos envolvendo a comunidade epilítica são relacionados principalmente a indicadores de condições tróficas e de saprobidade (Lobo et al. 1996, 2002, 2004a, b; Wetzel et al. 2002; Burliga et al. 2005; Salomoni et al. 2006; Hermany et al. 2006; Schneck et al. 2007; Salomoni \& Torgan 2008).

Em Minas Gerais o conhecimento sobre a flora diatomológica limita-se a estudos do fitoplâncton (Giani \& Leonardo 1988; Costa \& Torgan 1991; Soares et al. 2007), não havendo até o momento trabalhos envolvendo as comunidades epilíticas.

O presente trabalho tem por objetivo conhecer a composição taxonômica da comunidade de diatomáceas epilíticas do rio do Salto e a distribuição dos táxons nos períodos de chuva e estiagem, buscando contribuir para o incremento do conhecimento deste grupo em ambiente oligotrófico e ácido no Brasil. Amplia-se também as informações sobre a distribuição geográfica das Bacillariophyceae no estado de Minas Gerais.

\section{Material e métodos}

O Parque Estadual do Ibitipoca (PEI) localiza-se na serra da Mantiqueira, no município de Lima Duarte (MG), entre as coordenadas $21^{\circ} 40^{\prime}-21^{\circ} 44^{\prime} \mathrm{S}$ e $43^{\circ} 52^{\prime}$ $43^{\circ} 55^{\prime} \mathrm{W}$ (Fig. 1). Nele situa-se a nascente do rio do Salto, seu principal curso d'água. Da nascente ao limite sul do PEI, o rio corre por $5 \mathrm{Km}$, entre as cotas altimétricas de 1.650 e $1.050 \mathrm{~m}$. As amostragens de epilíton foram realizadas no leito rochoso do rio, em oito estações ao longo de seu curso superior, em janeiro e julho de 2004. Em cada estação foi delimitada uma área de $10 \mathrm{~m}^{2}$ onde foram sorteadas cinco posições. No total 80 amostras foram analisadas. Um coletor de epilíton especialmente confeccionado (Canani et al., 2010) foi usado para permitir o acesso ao leito rochoso e minimizar perdas durante a coleta. As amostras foram obtidas através de raspagem, utilizando-se escovas e uma seringa, e foram fixadas em solução de Lugol. A condutividade e a temperatura da água foram medidas em campo com termosalinômetro / oxímetro marca SC-TYSI mod.30 e o $\mathrm{pH}$ com potenciômetro DIGIMED mod. DMPA-PV. A análise para ortofosfato foi realizada no Laboratório de Hidrologia da Universidade Federal do Rio de Janeiro, segundo os protocolos citados em Paranhos (1996).

O material foi oxidado de acordo com o protocolo de Von Stoch (Tomas 1996), usando-se Naphrax como meio de montagem para as lâminas permanentes. As fotomicrografias foram obtidas em microscópio óptico Zeiss Axioplan, modelo Standard 18 e através de software Pro Plus (versão 4.5.0.19) acoplado a microscópio Olympus BH2. As amostras estão depositadas no Herbário do Museu Nacional do Rio de Janeiro (R).
O sistema de classificação e a terminologia usados seguiram Round et al. (1990). A identificação de táxons foi baseada em Frenguelli (1931-1933, 1941), Patrick \& Reimer (1966), Hein (1981), Round \& Mann (1981), Krammer \& Lange-Bertalot (1986, 1991), Vyverman \& Compère (1991), Lange-Bertalot \& Metzeltin (1996), Torgan et al. (1993), Costa (1995), Metzeltin \& Lange-Bertalot (1998), Moser (1999), Krammer (2000) e Sala et al. (2002).

\section{Resultados}

A água do rio do Salto apresentou valores de $\mathrm{pH}$ entre 4,2 e 5,5, e concentração de ortofosfato entre 1,37 e 10,98 $\mu \mathrm{g} . \mathrm{L}^{-1}$. A condutividade foi mais alta no período chuvoso (33-160 $\left.\mu \mathrm{S} . \mathrm{cm}^{-1}\right)$; na estiagem os valores ficaram entre $11,9-14,7 \mu \mathrm{S} . \mathrm{cm}^{-1}$. A temperatura da água no mês de janeiro variou entre $18,8-20^{\circ} \mathrm{C}$ (estação chuvosa) e entre 13,1-14,4 em julho (estação seca) (tabela 1).

Nesse estudo foram identificadas 15 espécies e duas variedades taxonômicas, distribuídas em duas classes, quatro ordens, oito famílias, descritas a seguir.

Fragilariophyceae Round 1990

Fragilariales Silva 1962

Fragilariaceae Greville 1833

Fragilariforma (J. Ralfs) D. M. Williams \& F. E. Round 1988

Fragilariforma stevensonii Metzeltin \& Lange-Bertalot, Icon. Diatomol. 18, p. 135. 2007.

Fig. 2-6

Valvas cruciformes, rômbico-lanceoladas, às vezes sigmóides ou subcirculares, fortemente infladas na região central; ápices arredondados, às vezes truncados ou rostrados; estrias transapicais paralelas tornando-se progressivamente curvas ou radiadas e mais densas em direção aos ápices; stermum parcialmente obliterado ou ausente. Compr. 10,2-32,5 $\mu \mathrm{m}$; larg. 8,3-15,2 $\mu \mathrm{m} ; 16-20$ estrias em $10 \mu \mathrm{m}$ no centro, ca. de 22 estrias em $10 \mu \mathrm{m}$ nas extremidades.

Material examinado: BRASIL. Minas Gerais: Lima Duarte, Parque Estadu al do Ibitipoca, rio do Salto, 27/I/2004, L.G.C. Canani \& U.J.Pereira s.n. (R 205103).

O material apresentou ampla variação quanto à forma das valvas e à distribuição e orientação das estrias: forma cruciforme com estrias paralelas, interrompidas ou não (fig. 2), forma sigmóide (fig. 3), forma elíptica com estrias paralelas no centro e radiadas nos ápices (fig. 4) ou forma subcirculare com estrias distribuídas desordenadamente (fig. 5). Em todos os indivíduos observados as estrias atingem a margem valvar na região central, que é expandida lateralmente.

A população encontrada no rio do Salto assemelha-se a Fragilariforma floridana sensu Williams. No protólogo de Fragilaria floridana, Hanna (1933) citou apenas as medidas 

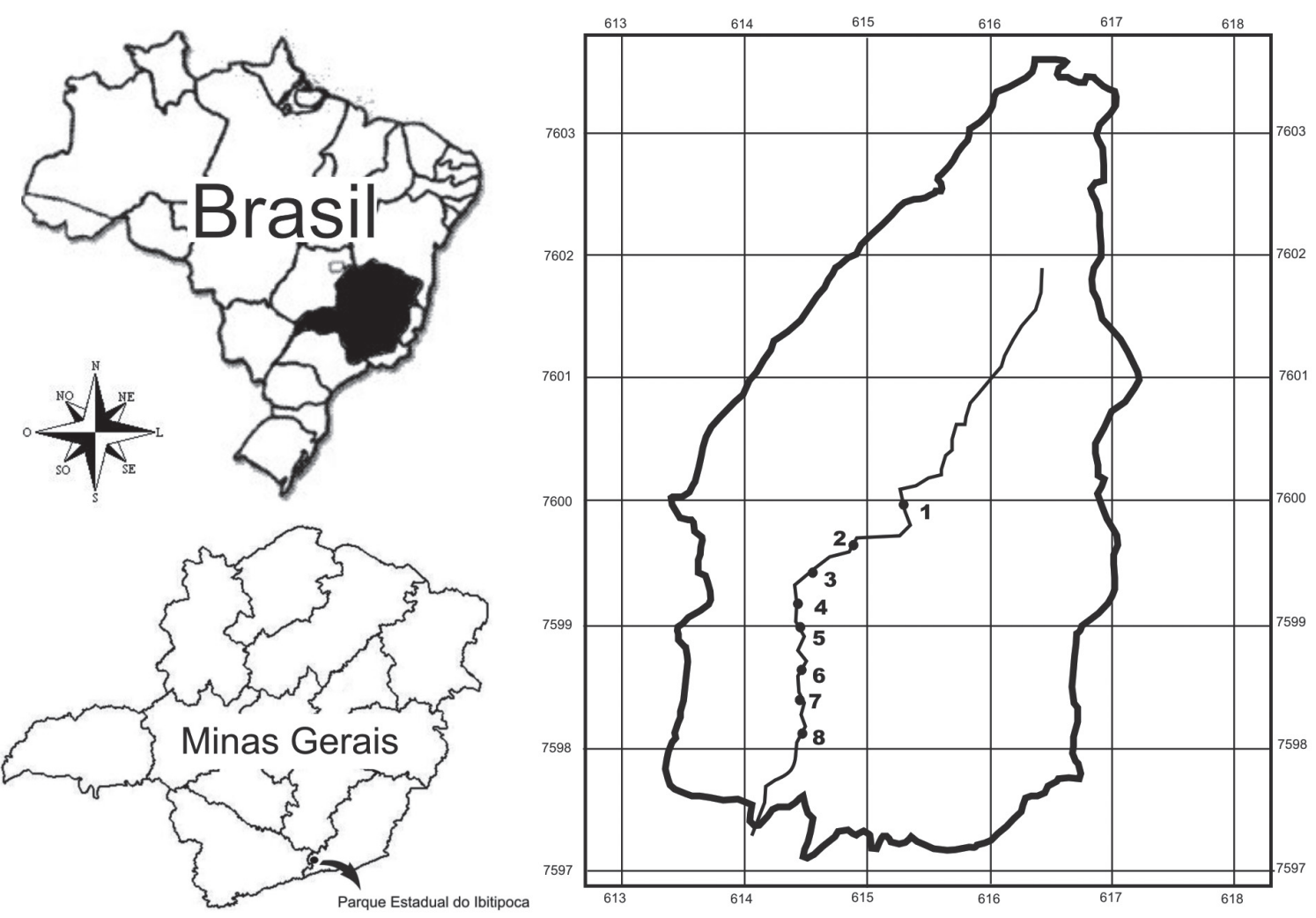

Figura 1. Localização geográfica do Parque Estadual do Ibitipoca, no estado de Minas Gerais, Brasil (coordenadas em UTM). No detalhe, localização das estações de amostragem no rio do Salto.

do holótipo e do parátipo, ilustrando apenas dois indivíduos e comentou que as estrias não atingiam a margem valvar na área central expandida. Patrick (1940) descreveu F. spinulosa, citando que as estrias atingiam a margem da valva na região central nesta espécie diferenciando-se de F. floridana. Entretanto, ao observar o material tipo de Hanna, Hein (1981) encontrou indivíduos com estrias alcançando as margens e outros não, não considerando esta característica como diagnóstica para a espécie. Através do acesso ao material tipo de Hanna (lâminas CAS\#345026 e CAS\#391058) confirmamos a ocorrência de indivíduos com ambas as características. Posteriormente, Williams (1990) transferiu o táxon F. floridana para o gênero Fragilariforma e o sinonimizou com Fragilaria spinulosa $\mathrm{Pa}$ trick. Metzeltin\&Lange-Bertalot (2007) desconsideram a sinonimização e além de transferirem Fragilaria spinulosa Patrick para o gênero Fragilariforma, descrevem algumas espécies novas para o gênero.

Tabela 1. Valores mínimos e máximos, média e desvio padrão de algumas variáveis abióticas aferidas no rio do Salto em janeiro (período de chuvas) e julho (período de estiagem) de 2004.

\begin{tabular}{|c|c|c|c|}
\hline Variáveis abióticas & & Período de chuvas & Período de estiagem \\
\hline \multirow{3}{*}{$\mathrm{pH}$} & Min-max & $4,5-6,8$ & $3,4-4,7$ \\
\hline & Média & 5 & 4,2 \\
\hline & DP & 0,84 & 0,551 \\
\hline \multirow{3}{*}{ Condutividade $(\mu \mathrm{S} / \mathrm{cm})$} & Min-max & $33-160$ & $11,9-14,7$ \\
\hline & Média & 127,62 & 12,57 \\
\hline & DP & 39,464 & 0,908 \\
\hline \multirow{3}{*}{ PSR $(\mu \mathrm{g} / \mathrm{L})$} & Min-max & $1,37-8,22$ & $3,08-10,98$ \\
\hline & Média & 5,08 & 5,49 \\
\hline & DP & 2,034 & 2,657 \\
\hline Temperatura $\left({ }^{\circ} \mathrm{C}\right)$ & Min-max & $18,8-22$ & $13,1-14,4$ \\
\hline
\end{tabular}



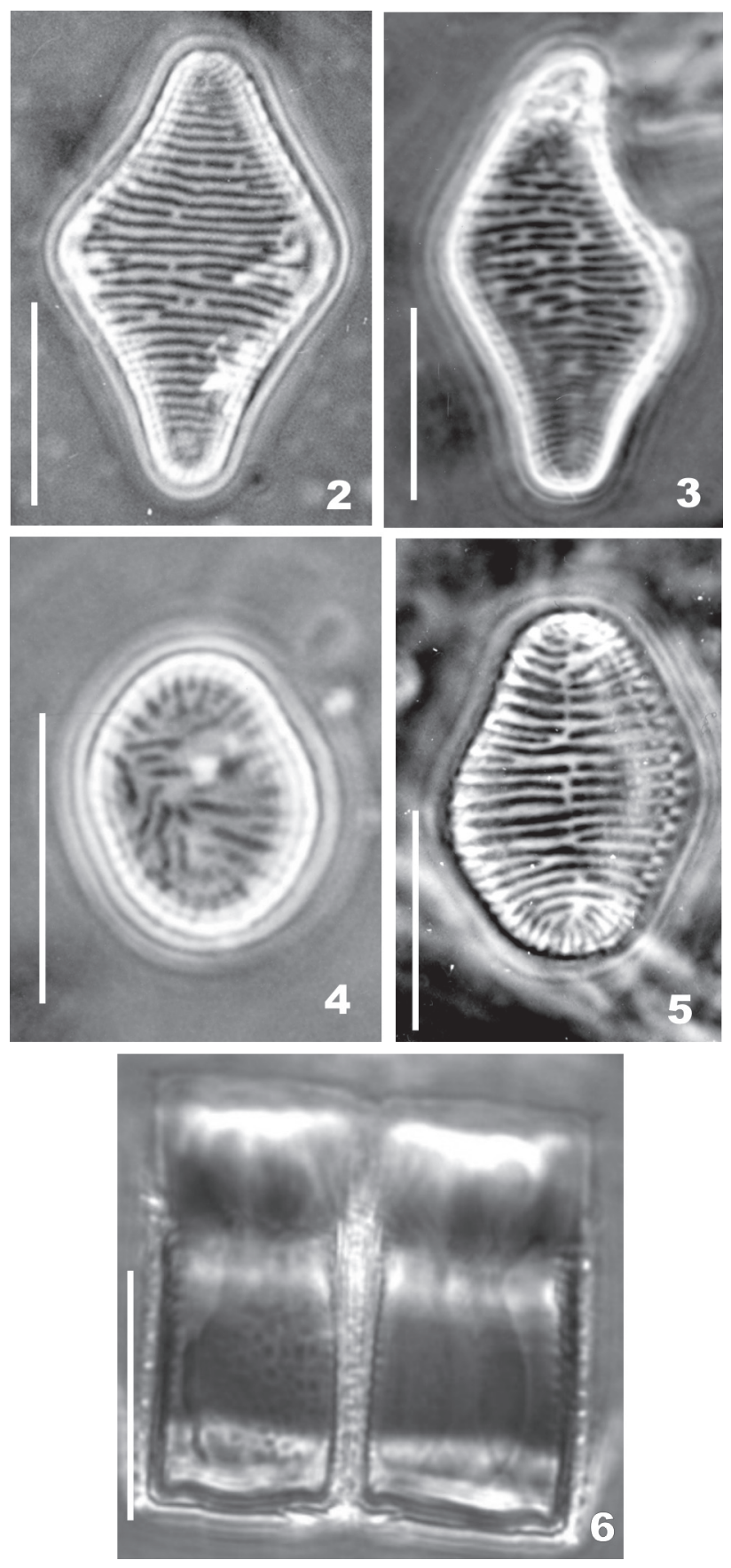

Figuras 2-6. Fragilariforma stevensonii. Barra $=10 \mu \mathrm{m}$.

Consideramos que a ausência ou presença de estrias alcançando a margem valvar seja um caráter relevante. Ao observarmos as descrições e ilustrações das novas espécies de Fragilariforma descritas por Metzeltin \& LangeBertalot (2007) os indivíduos encontrados no rio do Salto assemelharam-se à Fragilariforma stevensonii, em relação à densidade de estrias e ao contorno valvar. Ressaltamos que a população observada apresentou variabilidade morfológica não encontrada na literatura consultada. Ao assumir o fato das estrias irem ou não até a margem como característica importante na separação das espécies, implica em admitir que o material de Hanna (1933) possui mais do que uma espécie de Fragilariforma.

Bacillariophyceae Haeckel 1878

Eunotiales Silva 1962

Eunotiaceae Kützing 1844

Eunotia Ehrenberg 1837

Eunotia bidentula W. Smith, Syn. Brit. Diat. 2, p. 83. 1856. Fig. 7

Células isoladas; valvas com margem ventral reta a levemente côncava na região central, margem dorsal convexa com duas ondulações; ápices capitados arredondados; estrias radiadas; nódulos polares nítidos próximos aos ápices valvares. Compr. 22-27 $\mu \mathrm{m}$; larg. 5,5-8,5 $\mu \mathrm{m}$; $14-21$ estrias em $10 \mu \mathrm{m}$.

Material examinado: BRASIL. Minas Gerais: Lima Duarte, Parque Estadual do Ibitipoca, rio do Salto, 27/I/2004, epilíton, L.G.C. Canani \& U.J.Pereira, s.n. (R 205101, 205104); 28/I/2004, epilíton, L.G.C. Canani \& U.J.Pereira s.n. (R 205097, 205100); 21/VII/2004, epilíton, L.G.C. Canani \& U.J.Pereira s.n. (R 205105, 205106, 205107, 205108, 205109, 205111).

Eunotia exigua (Brébisson) Rabenhorst, Fl. Eur. Alg. I: 73. 1864.

Fig. 8

Células isoladas; valvas arqueadas, margem ventral moderada a fortemente côncava, margem dorsal fortemente convexa; ápices rostrados, voltados para a região dorsal; estrias paralelas; nódulos polares nos ápices valvares. Compr. 26,2-34,5 $\mu \mathrm{m}$; larg. 4-6 $\mu \mathrm{m} ; 20-22$ estrias em $10 \mu \mathrm{m}$.

Material examinado: BRASIL. Minas Gerais: Lima Duarte, Parque Estadual do Ibitipoca, rio do Salto, 21/VII/2004, epilíton, L.G.C. Canani \& U.J.Pereira s.n. (R 205107, 205108, 205109, 205111).

Os espécimes observados no rio do Salto apresentaram comprimento e largura superiores ao relatado por Petersen (1950), quais sejam: compr. 10-26; larg. 2,5-3,5, e número de estrias ligeiramente inferior, assemelhando-se às medidas citadas por Krammer \& Lange-Bertalot (1991).

Eunotia faba Ehrenberg, Ber. K. Akad. Wiss. Berlin: 45, fig 20. 1837.

Fig. 9-10

Células isoladas ou formando cadeias de dois a seis indivíduos; valvas lunadas a semicirculares, margem ventral reta a levemente côncava, margem dorsal convexa; ápices arredondados; estrias paralelas, com aréolas bem visíveis; nódulos polares nítidos, próximos aos ápices valvares. Compr. 17-71 $\mu \mathrm{m}$; larg. 5,5-12 $\mu \mathrm{m}$; 13-24 estrias em $10 \mu \mathrm{m}$.

Material examinado: BRASIL. Minas Gerais: Lima Duarte, Parque Estadual do Ibitipoca, rio do Salto, 27/I/2004, epilíton, L.G.C. Canani \& U.J.Pereira s.n. (R 205101, 205102, 
205103, 205104); 28/I/2004, epilíton, L.G.C. Canani \& U.J. Pereira s.n. (R 205097, 205098, 205099, 205100); 21/ VII/2004, epilíton, L.G.C. Canani \& U.J. Pereira s.n. (R 205105, 205106, 205107, 205108, 205109, 205110, 205111, 205112).

Os espécimes analisados apresentaram largura maior que $9 \mu \mathrm{m}$, valor máximo citado por Krammer \& LangeBertalot (1991).

Eunotia fallax A. Cleve, Kongl. Sven. Vet.-Akad. Handl. 21: 33, fig. 35. 1895.

Fig. 11

Células isoladas; valvas arqueadas, margem ventral levemente côncava na porção mediana, concavidade situada entre espessamentos bem marcados, margem dorsal levemente convexa; ápices capitados voltados para a região dorsal; estrias paralelas; nódulos polares nos ápices valvares, deslocados em direção à margem dorsal. Compr. 26,4-38,4 $\mu \mathrm{m}$; larg. 4-4,8 $\mu \mathrm{m}$; 13-17 estrias em $10 \mu \mathrm{m}$.

Material examinado: BRASIL. Minas Gerais: Lima Duarte, Parque Estadual do Ibitipoca, rio do Salto, 27/I/2004, epilíton, L.G.C. Canani \& U.J. Pereira s.n. (R 205101, 205103); 28/I/2004, epilíton, L.G.C. Canani \& U.J. Pereira (R 205100).

Eunotia intermedia (Krasske ex Hustedt) M. Nörpel \& Lange-Bertalot, Bibl. Diatomol. 27, p.32. 1993.

Fig. 12

Células isoladas; valvas lineares, margem ventral reta a levemente côncava, margem dorsal fracamente convexa; ápices arredondados; estrias paralelas; nódulos polares pouco visíveis nos ápices. Compr. 12,8-15,6 $\mu \mathrm{m}$; larg. 2,6-3 $\mu \mathrm{m} ; 18-20$ estrias em $10 \mu \mathrm{m}$.

Material examinado: BRASIL. Minas Gerais: Lima Duarte, Parque Estadual do Ibitipoca, rio do Salto, 27/I/2004, epilíton, L.G.C. Canani \& U.J. Pereira s.n. (R 205101, 205102, 205103, 205104); 28/I/2004, epilíton, L.G.C. Canani \& U.J. Pereira s.n. (R 205097, 205098, 205099, 205100); 21/VII/2004, epilíton, L.G.C. Canani \& U.J. Pereira s.n. (R 205105, 205106, 205107, 205108, 205110, 205112).

Os exemplares apresentaram menores dimensões (comp. 14-45 $\mu \mathrm{m}$; larg. 3,5-5 $\mu \mathrm{m}$ ) do que as referidas por Krammer \& Lange-Bertalot (1991) para esta espécie.

Eunotia pirarucu Metzeltin \& Lange-Bertalot, Icon. Diatomol. 5, p. 332, fig. 49:1-3. 1998.

Fig. 13

Células isoladas; valvas lunadas, margem ventral côncava, margem dorsal convexa; ápices amplamente arredondados; estrias irregularmente distribuídas, às vezes interrompidas junto à margem ventral, paralelas no centro a radiadas nos ápices; nódulos polares nítidos, distanciados dos ápices valvares. Compr. 89,5-114 $\mu \mathrm{m}$; larg. 25-33,9 $\mu \mathrm{m}$; 4-6 estrias (no centro) em $10 \mu \mathrm{m}$.

Material examinado: BRASIL. Minas Gerais: Lima Duarte, Parque Estadual do Ibitipoca, rio do Salto, 21/
VII/2004, epilíton, L.G.C. Canani \& U.J. Pereira s.n. (R 205106, 205107, 205108, 205111).

O material analisado possui dimensões menores do que as mínimas citadas na descrição da espécie (compr. 135-160 $\mu \mathrm{m}$; larg. 32-40 $\mu \mathrm{m}$ ) por Metzeltin \& Lange-Bertalot (1998).

Eunotia pseudoindica Frenguelli, Rev. Museo la Plata, sér. Bot. 3: 307, 1941.

Fig. 14

Células isoladas; valvas arqueadas; margem ventral côncava, reta nos ápices, margem dorsal convexa, reta nos ápices; ápices levemente cuneados, destacados da valva; estrias paralelas; nódulos polares próximos aos ápices valvares. Compr. 71-137 $\mu \mathrm{m}$; larg. 9-11 $\mu \mathrm{m}$; 10-14 estrias em $10 \mu \mathrm{m}$.

Material examinado: BRASIL. Minas Gerais: Lima Duarte, Parque Estadual do Ibitipoca, rio do Salto, 21/VII/2004, epilíton, L.G.C. Canani \& U.J. Pereira s.n. (R 205108).

Eunotia serra Ehrenberg, Ber. K. Akad. Wiss. Berlin: 45. 1837.

Fig. 15

Células isoladas; valvas arqueadas, margem ventral côncava, margem dorsal convexa com onze ondulações; ápices atenuadotruncados; estrias paralelas; nódulos polares indistintos. Compr. 63-96 $\mu \mathrm{m}$; larg. 9-11 $\mu \mathrm{m}$; 11-14 estrias em $10 \mu \mathrm{m}$.

Material examinado: BRASIL. Minas Gerais: Lima Duarte, Parque Estadual do Ibitipoca, rio do Salto, 27/I/2004, epilíton, L.G.C. Canani \& U.J. Pereira s.n. (R 205103); 21/ VII/2004, epilíton, L.G.C. Canani \& U.J. Pereira (R 205105, 205107, 205108, 205109).

Cymbellales Mann 1990

Cymbellaceae Greville 1833

\section{Encyonema Kützing 1833}

Encyonema hebridicum Grunow ex Cleve in Cleve \& Möller, Acta Soc. Fauna et Flora Fenn. 8(2):48, pl. 2: figs. 16,17. 1891.

Fig. 16

Células isoladas; valva semilanceolada, margem ventral levemente convexa, margem dorsal convexa; ápices apiculados; estrias paralelas; rafe deslocada para a porção mais ventral da valva; área central semicircular a elíptica. Compr. $35 \mu \mathrm{m}$; larg. $7 \mu \mathrm{m}$; 12 estrias em $10 \mu \mathrm{m}$ na região dorsal; 11 estrias em $10 \mu \mathrm{m}$ na região ventral.

Material examinado: BRASIL. Minas Gerais: Lima Duarte, Parque Estadual do Ibitipoca, rio do Salto, 27/I/2004, epilíton, L.G.C. Canani \& U.J. Pereira s.n. (R 205104); 21/ VII/2004, epilíton, L.G.C. Canani \& U.J. Pereira s.n. (R 205106, 205107, 205108, 205109, 205111).

Naviculales Bessey 1907

Amphipleuraceae Grunow 1862 

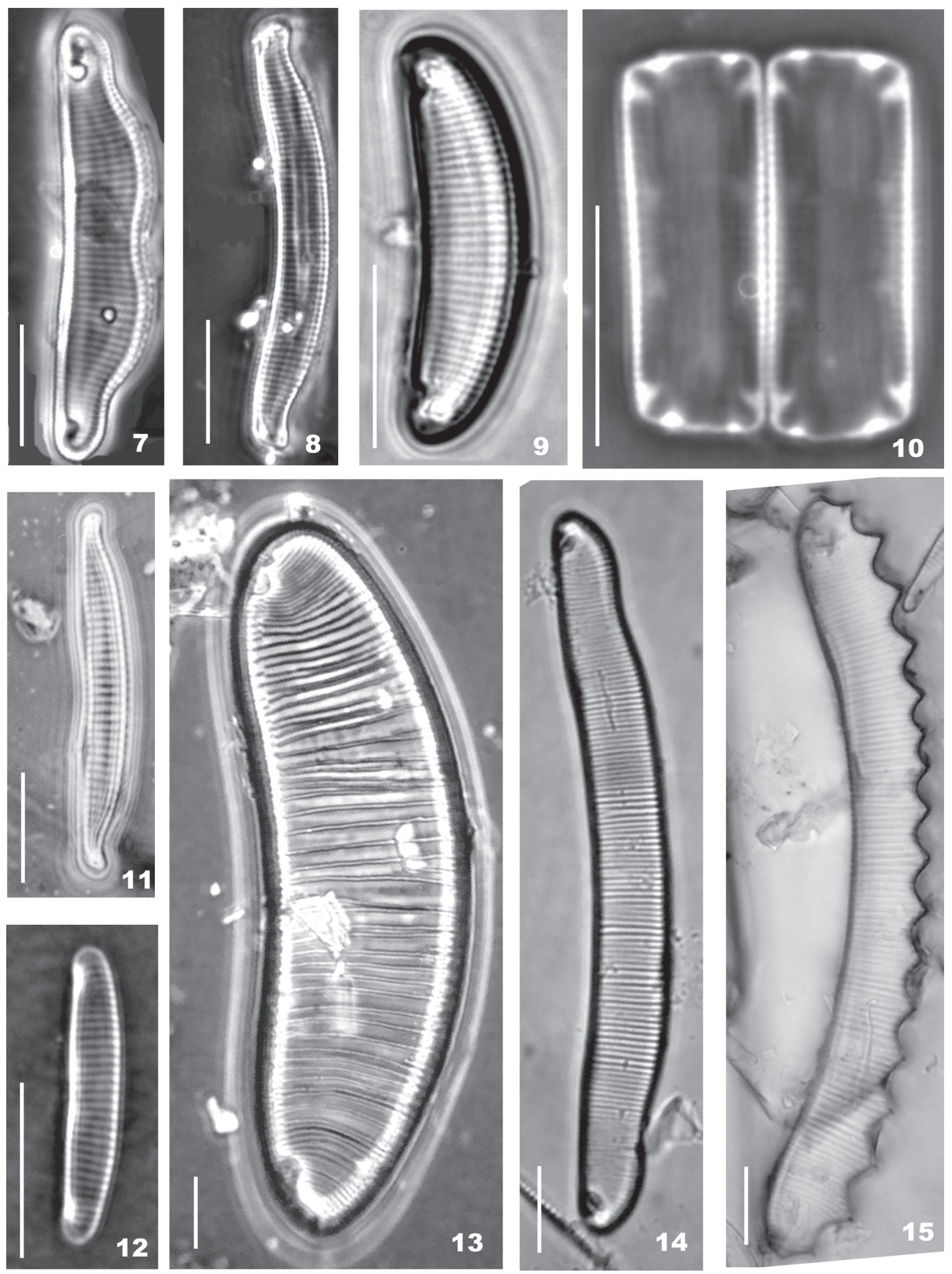

Figura 7. Eunotia bidentula; 8. E. exigua; 9-10. E. faba; 11. E. fallax; 12. E. intermedia; 13. E. pirarucu; 14. E. pseudoindica; 15. E. serra. Barra $=10 \mu \mathrm{m}$. 
Frustulia Rabenhorst 1853

Frustulia crassinervia (Brébisson) Costa, Iheringia Ser. Bot. 46: 88. 1995.

Fig. 17

Células isoladas; valvas lanceoladas a elíptico-lanceoladas; margens levemente onduladas; ápices sub-rostrados; rafe filiforme reta entre duas costelas longitudinais; nódulo central constrito na região mediana. Compr. 50-54 $\mu \mathrm{m}$; larg. 10,8-16,2 $\mu \mathrm{m} ; 33-34$ estrias transapicais em $10 \mu \mathrm{m} ; 25$ estrias longitudinais em $10 \mu \mathrm{m}$.

Material examinado: BRASIL. Minas Gerais: Lima Duarte, Parque Estadual do Ibitipoca, rio do Salto, 27/I/2004, epilíton, L.G.C. Canani \& U.J. Pereira (R 205103); 21/ VII/2004, epilíton, L.G.C. Canani \& U.J. Pereira (R 205105, 205107, 205108, 205109).

Frustulia saxonica Rabenhorst, Süssw. Diat. 50, pl 7, fig. 1. 1853.

Fig. 18

Células isoladas, valvas lanceoladas; ápices sub-rostrados; rafe filiforme reta, nódulo central constrito na região mediana. Compr. 38,3-90; larg. 10,8-16,2 $\mu \mathrm{m}$; 33-34 estrias transapicais em $10 \mu \mathrm{m} ; 27-30$ estrias longitudinais em $10 \mu \mathrm{m}$.

Material examinado: BRASIL. Minas Gerais: Lima Duarte, Parque Estadual do Ibitipoca, rio do Salto, 27/I/2004, epilíton, L.G.C. Canani \& U.J. Pereira s.n. (R 205101, 205102, 205103, 205104); 28/I/2004, epilíton, L.G.C. Canani \& U.J. Pereira s.n. (R 205097, 205098, 205100); 21/ VII/2004, epilíton, L.G.C. Canani \& U.J. Pereira s.n. (R 205105, 205106, 205107, 205108, 205109).

Brachysiraceae D.G. Mann 1990

\section{Brachysira Kützing 1836}

Brachysira brebissonii R. Ross 1986 in Hartley, J. Mar. Biol. Ass. U.K. 66: 607. 1986.

Fig. 19

Células isoladas; valvas lanceoladas; ápices atenuadoarredondados; área central rômbica; estrias formadas por aréolas alongadas transapicalmente; rafe filiforme reta. Compr. 25-35 $\mu \mathrm{m}$; larg. 7-8 $\mu \mathrm{m}$; 23 estrias em $10 \mu \mathrm{m}$.

Material examinado: BRASIL. Minas Gerais: Lima Duarte, Parque Estadual do Ibitipoca, rio do Salto, 21/VII/2004, epilíton L.G.C. Canani \& U.J. Pereira s.n. (R 205110).

Brachysira serians var. rostrata (Krasske) Lange-Bertalot \& Moser, Bibl. Diatomol. 29: 62, fig. 35:4-6. 1994.

Fig. 20

Células isoladas; valvas lanceoladas; ápices alongados rostrados; área central arredondada; estrias formadas por aréolas alongadas transapicalmente; rafe filiforme reta.

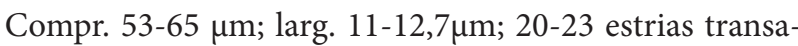
picais em $10 \mu \mathrm{m}$.

Material examinado: BRASIL. Minas Gerais: Lima Duarte, Parque Estadual do Ibitipoca, rio do Salto, 21/ VII/2004, epilíton, L.G.C. Canani \& U.J. Pereira s.n. (R 205110).

Os espécimes observados conferem com os ilustrados por Krasske (1948) na obra original. O material foi coletado em musgos no estado São Paulo tendo sido considerado pelo autor como indicador de águas de baixo $\mathrm{pH}$ e ricas em ácidos húmicos.

Brachysira subrostrata Lange-Bertalot \& Moser foi descrita pelos autores a partir da observação do material de Krasske. Esta espécie diferencia-se de B. serians var. rostrata por possuir ápices menos rostrados e valvas lanceoladas a rômbico-lanceoladas.

Naviculaceae Kützing 1844

Nupela Vyverman \& Compère 1991

Nupela giluwensis Vyverman \& Compère, Diatom Res. 6(1), p. 175-179. 1991.

Fig. 21

Células isoladas; valvas elípticas levemente curvadas em relação ao eixo apical; ápices capitados; área central retangular, extremidades distais da rafe curvadas lateralmente, para a mesma direção. Compr. 10-18,5 $\mu$ m; larg. 3-4,5 $\mu$ m; estrias inconspícuas.

Material examinado: BRASIL. Minas Gerais: Lima Duarte, Parque Estadual do Ibitipoca, rio do Salto, 27/I/2004, epilíton, L.G.C. Canani \& U.J. Pereira s.n. (R 205101); 28/I/2004, epilíton, L.G.C. Canani \& U.J. Pereira s.n. (R 205097); 21/VII/2004, epilíton, L.G.C. Canani \& U.J. Pereira s.n. (R 205105, 205106, 205108, 205110, 205112).

Pinnulariaceae Mann 1990

Pinnularia Ehrenberg 1843

Pinnularia cf. microstauron var. rostrata Krammer, Diat. Eur. 1, p.74, figs.51:8-18. 2000.

Fig. 22

Célula isolada; valvas lineares; ápices subcapitados; área central retangular; estrias radiadas no centro, tornando-se progressivamente convergentes em direção aos ápices; extremidades distais da rafe em forma de gancho; Compr. $45 \mu \mathrm{m}$; larg. 9,8 $\mu \mathrm{m} ; 11$ estrias em $10 \mu \mathrm{m}$.

Material examinado: BRASIL. Minas Gerais: Lima Duarte, Parque Estadual do Ibitipoca, rio do Salto, 27/I/2004, epilíton, L.G.C. Canani \& U.J. Pereira s.n. (R 205104); 21/ VII/2004, epilíton, L.G.C. Canani \& U.J. Pereira s.n. (R 205106, 205108, 205109, 205111).

As medidas do espécime observado concordam com aquelas descritas para P. microstauron (Ehrenberg) Cleve, a 

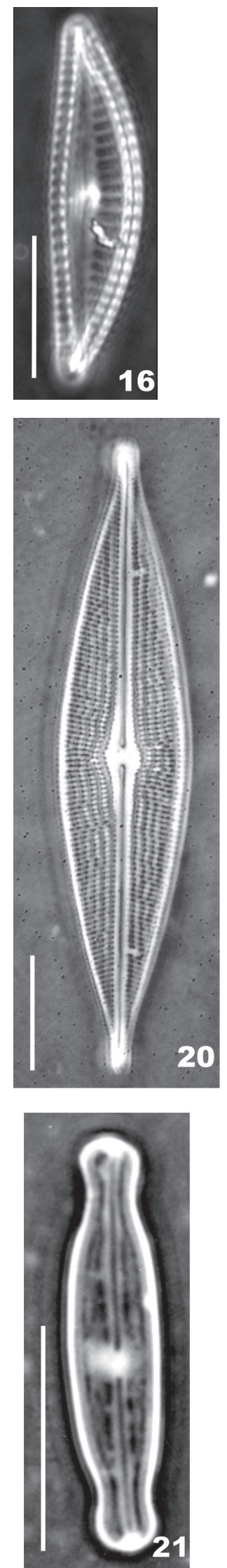
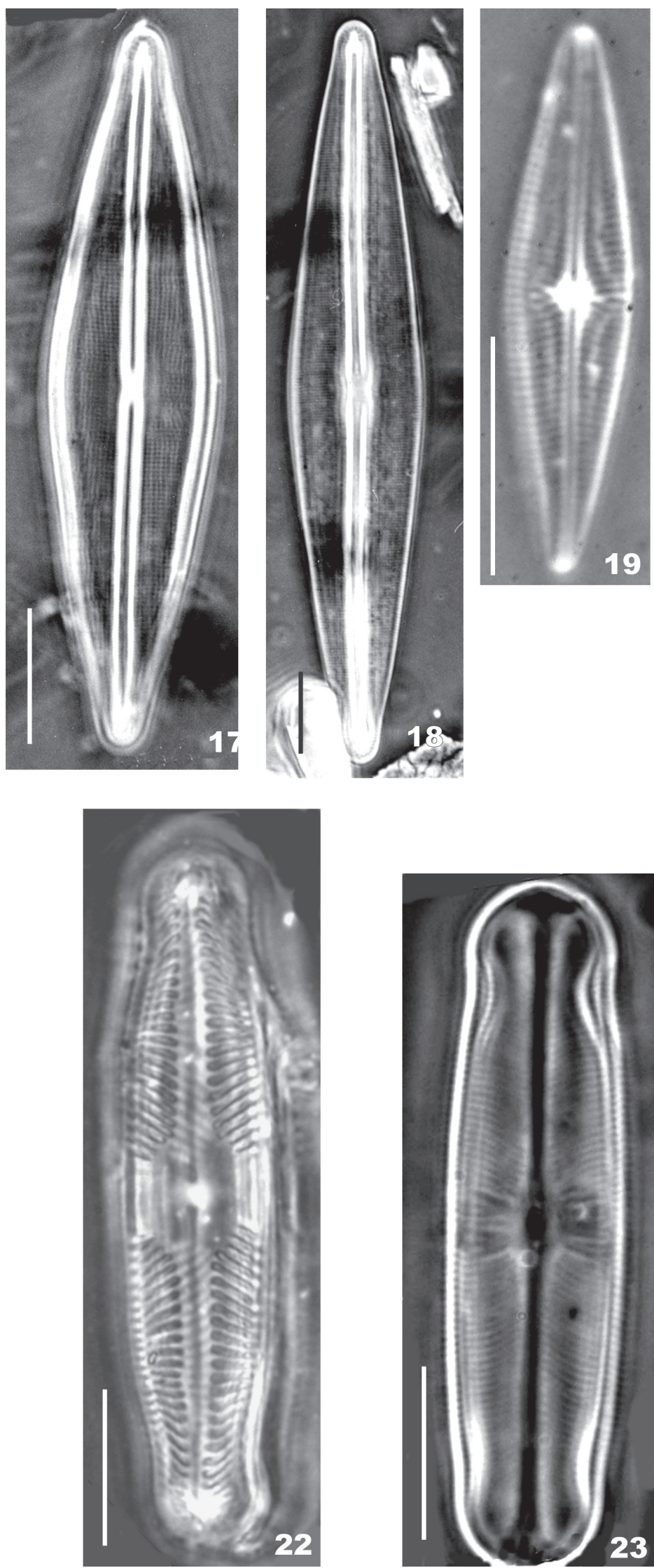

Figura 16. Encyonema hebridicum; 17. F. crassinervia; 18. F. saxonica; 19. Brachysira brebissoni; 20. B. serians var. rostrata; 21. Nupela giluwensis; 22. Pinnularia microstauron var. rostrata; 23 Sellaphora blackfordensis..Barra $=10 \mu \mathrm{m}$. 
partir de material coletado no Rio de Janeiro. Morfologicamente assemelha-se a $P$. microstauron var. rostrata, porém a largura encontrada $(9,8 \mu \mathrm{m})$ foi maior do que aquela citada por Krammer $(6-7 \mu \mathrm{m})$ para a variedade, motivo pelo qual foi mantido em conferatur. O táxon também aproxima-se morfologicamente de P. subanglica, porém esta apresenta área axial mais estreita.

Sellaphoraceae Mereschkowsky 1902

Sellaphora Mereschkowsky 1902

Sellaphora blackfordensis Mann \& Droop, Phycologia 43(4), p. 476, figs. 4g-I, 19, 33-37. 2004.

Fig. 23

Células isoladas; valvas lineares; ápices sub-capitados; barras polares presentes, área axial estreita, área central em forma de gravata-borboleta, com contorno irregular devido à presença de estrias longas e curtas alternadas; estrias de radiadas no centro a paralelas nos ápices. Compr. $40 \mu \mathrm{m}$; larg. 9,6-10,3 $\mu \mathrm{m} ; 20-22$ estrias em $10 \mu \mathrm{m}$.

Material examinado: BRASIL. Minas Gerais: Lima Duarte, Parque Estadual do Ibitipoca, rio do Salto, 21/ VII/2004, epilíton, L.G.C. Canani \& U.J. Pereira (R 205109).

O gênero Sellaphora passou por recentes revisões e várias espécies novas foram propostas por Mann et al. (2008). Os autores consideraram importantes as características morfológicas (largura da área axial, forma da área central, nitidez do nódulo central e a presença de barras polares) para separar os grupos de espécies, não levando em conta a forma valvar. O indivíduo encontrado no rio do Salto apresenta barras polares, não possui área central circular e seu nódulo central é pouco nítido, características que são compartilhadas pelas espécies do grupo "pupula". S. blackfordensis diferencia-se de S. capitata Mann \& McDonald, por ser mais larga e pela tendência de a segunda ser mais fortemente capitada (Mann \& Droop 2004).

\section{Discussão}

Entre os 17 taxa específicos e infraespecíficos identificados (distribuídos em duas classes, duas ordens, oito famílias e nove gêneros), duas já haviam sido citados para o estado de Minas Gerais: Frustulia crassinervia e F. saxonica, no lago dos Manacás, município de Juiz de Fora (Costa \& Torgan, 1991). Os demais 15 táxons são registros pioneiros para o Estado.

O gênero mais representativo foi Eunotia, com oito espécies. E. faba esteve presente em todas as estações amostradas nos períodos de chuva e estiagem. As espécies com freqüencia acima de 50\% foram Eunotia bidentula, E. intermedia e Frustulia saxonica. A preponderância do gênero Eunotia nas amostras é explicada pelo baixo $\mathrm{pH}$ do ambiente estudado, sendo este gênero descrito como acidófilo por Round et al. (1990) e Metzeltin \& Lange-Bertalot (1998). Por outro lado, Brachisira brebissoni, B. serians var. rostrata, Eunotia pseudoindica e Sellaphora blackfordensis foram encontradas em uma única estação, apenas no período de estiagem. Em estudos onde as diatomáceas foram relacionadas ao nível trófico do rio, Sellaphora pupula sensu lato foi abundante em ambientes médio e fortemente poluídos (Lobo et al. 1996, 2004b; Salomoni et al. 2006), o que provavelmente pode explicar sua baixa freqüência no rio do Salto. Frustulia crassinervia, com freqüência de $37,5 \%$ no rio do Salto, foi considerada por Wetzel et al. (2002) como indicadora de águas oligotróficas e de baixo $\mathrm{pH}$.

De maneira geral, a riqueza de diatomáceas foi maior na estiagem (tabela 2), provavelmente devido ao menor distúrbio nesse período, permitindo que mais organismos permanecessem aderidos ao biofilme epilítico. Segundo Schneck et al. (2008), a riqueza de diatomáceas em rios de altitude comumente apresenta-se menor que a observada em sistemas de planície, provavelmente em decorrência de maior correnteza, temperaturas mais baixas e menor concentração de nutrientes. No rio do Salto também foi encontrado um número menor de táxons quando comparado a estudos realizados em trechos médio e baixo de rios, ricos em nutrientes. Hermany et al. (2006) observaram 147 espécies em um riacho na região hidrográfica do Guaíba e Salomoni et al. (2006) registraram 166 táxons no rio Gravataí, ambos no Rio Grande do Sul.

Eunotia exigua, E. fallax, E. intermedia, E. pseudoindica, E. serra e Brachysira serians foram encontradas no plâncton e perifíton de ambientes lóticos brasileiros (Brassac and Ludwig, 2003; Landucci and Ludwig, 2005; Procopiak et. al. 2006; Aprile and Mera, 2007; Ferrari and Ludwig, 2007; Ferrari et. al. 2007; Tremarin et. al. 2008), no entanto ainda não haviam sido registradas no epilíton. Fragilariforma stevensonii, Eunotia pirarucu, Sellaphora blackfordensis, Nupela giluwensis são primeiras ocorrências para o Brasil.

As águas do curso superior do rio do Salto, objeto do presente estudo, enquadram-se na classe "Especial" (Resolução no 16/96 do COPAM/MG), ou seja, águas destinadas ao abastecimento doméstico sem prévia ou simples desinfecção e à preservação do equilíbrio natural das comunidades aquáticas. Assim sendo, o conhecimento da flora de diatomáceas encontrada neste rio constitui-se numa referência para sistemas com estas características em outras regiões do Brasil.

\section{Agradecimentos}

Ao Instituto Estadual de Florestas de Minas Gerais (IEF) pelo apoio logístico no Parque Estadual do Ibitipoca, ao Conselho Nacional de Desenvolvimento Científico e Tecnológico (CNPq) pela concessão de bolsas de mestrado e doutorado ao primeiro autor e bolsas de Produtividade à Pesquisa aos demais autores. Ao Museu Nacional da Universidade Federal do Rio de Janeiro e à Fundação Zoobotânica 
Tabela 2. Riqueza e freqüência relativa dos táxons identificados por estação de amostragem no período de chuva (jan /2004) e estiagem (jul/2004) no rio do Salto, Minas Gerais.

\begin{tabular}{|c|c|c|c|c|c|c|c|c|c|c|c|c|c|c|c|c|c|}
\hline \multirow{2}{*}{$\begin{array}{l}\text { Períodos } \\
\text { Taxa/Estações }\end{array}$} & \multicolumn{8}{|c|}{ Chuva } & \multicolumn{8}{|c|}{ Estiagem } & \multirow{2}{*}{$\begin{array}{l}\text { Freqüência } \\
\text { relativa (\%) }\end{array}$} \\
\hline & 1 & 2 & 3 & 4 & 5 & 6 & 7 & 8 & 1 & 2 & 3 & 4 & 5 & 6 & 7 & 8 & \\
\hline Brachysira brebisonii & & & & & & & & & & & & & & & $\mathrm{X}$ & & 6,25 \\
\hline Brachysira serians var. rostrata & & & & & & & & & & & & & & & $\mathrm{X}$ & & 6,25 \\
\hline Encyonema hebridicum & & & & & & & $\mathrm{X}$ & $\mathrm{X}$ & & $\mathrm{X}$ & $\mathrm{X}$ & $\mathrm{X}$ & $\mathrm{X}$ & & $\mathrm{X}$ & & 43,75 \\
\hline Eunotia bidentula & $\mathrm{X}$ & & & $\mathrm{X}$ & $\mathrm{X}$ & & & $\mathrm{X}$ & $\mathrm{X}$ & $\mathrm{X}$ & $\mathrm{X}$ & $\mathrm{X}$ & $\mathrm{X}$ & & $\mathrm{X}$ & & 62,5 \\
\hline Eunotia exigua & & & & & & & & & & & $\mathrm{X}$ & $\mathrm{X}$ & $\mathrm{X}$ & & $\mathrm{X}$ & & 25 \\
\hline Eunotia faba & $\mathrm{X}$ & $\mathrm{X}$ & $\mathrm{X}$ & $\mathrm{X}$ & $\mathrm{X}$ & $\mathrm{X}$ & $\mathrm{X}$ & $\mathrm{X}$ & $\mathrm{X}$ & $\mathrm{X}$ & $\mathrm{X}$ & $\mathrm{X}$ & $\mathrm{X}$ & $\mathrm{X}$ & $\mathrm{X}$ & $\mathrm{X}$ & 100 \\
\hline Eunotia fallax & & & & $\mathrm{X}$ & $\mathrm{X}$ & & $\mathrm{X}$ & & & & & & & & & & 18,75 \\
\hline Eunotia intermedia & $\mathrm{X}$ & $\mathrm{X}$ & $\mathrm{X}$ & $\mathrm{X}$ & $\mathrm{X}$ & $\mathrm{X}$ & $\mathrm{X}$ & $\mathrm{X}$ & $\mathrm{X}$ & $\mathrm{X}$ & $\mathrm{X}$ & $\mathrm{X}$ & $\mathrm{X}$ & & $\mathrm{X}$ & & 87,5 \\
\hline Eunotia pirarucu & & & & & & & & & & $\mathrm{X}$ & $\mathrm{X}$ & $\mathrm{X}$ & & & $\mathrm{X}$ & & 25 \\
\hline Eunotia pseudoindica & & & & & & & & & & & & $\mathrm{X}$ & & & & & 6,25 \\
\hline Eunotia serra & & & & & & & $\mathrm{X}$ & & $\mathrm{X}$ & & $\mathrm{X}$ & $\mathrm{X}$ & $\mathrm{X}$ & & & & 31,25 \\
\hline Fragilariforma stevensonii & & & & & & & $\mathrm{X}$ & & & & & & & & $\mathrm{X}$ & & 12,5 \\
\hline Frustulia crassinervia & & & & & & & $\mathrm{X}$ & & $\mathrm{X}$ & $\mathrm{X}$ & $\mathrm{X}$ & $\mathrm{X}$ & $\mathrm{X}$ & & & & 37,5 \\
\hline Frustulia saxonica & $\mathrm{X}$ & $\mathrm{X}$ & & $\mathrm{X}$ & $\mathrm{X}$ & $\mathrm{X}$ & $\mathrm{X}$ & $\mathrm{x}$ & $\mathrm{X}$ & $\mathrm{X}$ & $\mathrm{X}$ & $\mathrm{X}$ & $\mathrm{X}$ & & & & 75 \\
\hline Nupela giluwensis & $\mathrm{X}$ & & & & $\mathrm{X}$ & & & & $\mathrm{X}$ & $\mathrm{X}$ & $\mathrm{X}$ & & $\mathrm{X}$ & & $\mathrm{X}$ & & 43,75 \\
\hline Pinnularia microstauron var. rostrata & & & & & & & & $\mathrm{x}$ & & $\mathrm{X}$ & $\mathrm{X}$ & & $\mathrm{X}$ & & $\mathrm{X}$ & & 31,25 \\
\hline Sellaphora blackfordensis & & & & & & & & & & & & & $\mathrm{X}$ & & & & 6,25 \\
\hline Riqueza & 5 & 3 & 2 & 5 & 6 & 3 & 8 & 6 & 7 & 11 & 11 & 10 & 11 & 1 & 11 & 1 & \\
\hline
\end{tabular}

do Rio Grande do Sul pelo apoio logístico. Ao Programa de Pós-Gradução em Botânica da Universidade Federal do Rio Grande do Sul, pela oportunidade de dar continuidade aos estudos nesta área.

\section{Referências bibliográficas}

Aprile, F.M. \& Mera, P.A.S. 2007. Fitoplâncton e Fitoperifíton de um Rio de Águas Pretas da Amazônia Periférica do Norte, Brasil. Brazilian Journal of Aquatic Science and Technology 11: 1-14.

Brassac, N.M \& Ludwig T.A.V. 2003. Fragilariaceae (Bacillariophyceae) de rios da bacia do Iguaçu, Estado do Paraná, Brasil. Revista Brasileira de Botânica 26: 311-318.

Burliga, A.L.; Torgan, L.C.; Nobrega, E.A.; Beaumord, A.C.; Costa, C.O. \& Wamauti, D.V.2005. Diatomáceas epilíticas do rio Itajaí-Mirim, Santa Catarina, Brasil. Acta Scientiarum, Biological Sciences 27: 415-421.

Canani, L.G.C.; Torgan, L.C. \& Menezes, M. 2010. Gadget for Epilithic Microalgae Sampling (GEMS). Brazilian Journal of Biology 70: 289-291.

Cholnoky, B.J. 1957. Über die Diatomeenflora einiger Gewaesseer in den Magalies-Bergen nahe Rustenburg (Transvaal). Botaniska Notiser 110: 325-362.

COPAM 1996. Instituto Mineiro de Gestão das Águas. Deliberação Normativa COPAM 16/96. disponível em: http://www.siam.mg.gov. $\mathrm{br} / \mathrm{sla} /$ download.pdf?idNorma $=113$.

Costa, J.C.F. 1995. Diatomáceas (Bacillariophyceae) da Reserva Biológica de Poço das Antas, município de Silva Jardim, Rio de Janeiro, Brasil. Iheringia, Série Botânica 46: 57-144.

Costa, J.C.F. \& Torgan, L.C. 1991. Análise taxonômica de diatomáceas (Bacillariophyceae) do lago da Universidade Federal de Juiz de Fora, Minas Gerais, Brasil. Iheringia, Série Botânica 41: 47-81.

Ferrari, F. \& Ludwig, T.A.V. 2007. Coscinodiscophyceae, Fragilariophyceae e Bacillariophyceae (Achnanthales) dos rios Ivaí, São João e dos Patos, bacia hidrográfica do rio Ivaí, município de Prudentópolis, PR, Brasil. Acta Botanica Brasilica 21: 421-441.

Ferrari, F.; Procopiak, L.K; Alencar, Y.B \& Ludwig, T.A.V. 2007. Eunotiaceae (Bacillariophyceae) em igarapés da Amazônia Central, Manaus e Presidente Figueiredo, Brasil. Acta Amazonica 37: 1-16.

Frenguelli, J. 1931-1933. Contribuciones al conocimiento de las Diatomeas Argentinas. VII Diatomeas de la Región de los Esteros del Yberá (en la Provincia de Corrientes). Anales del Museo Nacional del Historia Natural 37: 365-476.

Frenguelli, J. 1941. Diatomeas del Rio Río de la Plata. Revista del Museo de La Plata (Nueva Serie) 3: 213-334.

Giani, A. \& Leonardo, I.M. 1988. Distribuição vertical de algas fitoplanctônicas no reservatório da Pampulha (Belo Horizonte, MG). Acta Limnologica Brasiliensia 2: 387-404.

Hanna, G.D. 1933. Diatoms of the Florida peat deposits. Florida State Geological Survey, $2^{\text {rd }}$ and $24^{\text {th }}$ annual report, 1930-1932. Pp. 57-64.

Hein, M.K. 1981. Variability in the diatom Fragilaria floridana Hanna. Proceedings of the Iowa Academy of Science 88: 79-81.

Hermany, G.; Schwarzbold, A.; Lobo, E.A. \& Oliveira, M.A. 2006. Ecology of the epilithic diatom community in a low-order stream system of Guaiba hydrographical region: subsidies to the environmental monitoring of southern Brazilian aquatic systems. Acta Limnologica Brasilensia 18: 9-27.

Kociolek, J.P. \& Stoermer, E.F. 2009. Oligotrophy: the forgotten end of an ecological spectrum. Acta Botanica Croatica 68: 465-472.

Krammer, K. 2000. The Genus Pinnularia. In: Lange Bertalot (Ed.). Diatoms of Europe, vol. 1, Ruggell, A.R.G. Gartner Verlag K.G.

Krammer, K. \& Lange-Bertalot, H. 1986. Susswasserflora von Mitteleuropa. Bacillariophyceae $2 / 1$. Stuttgart, G. Fisher.

Krammer, K. \& Lange-Bertalot, H. 1991. Susswasserflora von Mitteleuropa. Bacillariophyceae $2 / 3$. Stuttgart, G. Fisher.

Krasske, G. 1948. Diatomeen Tropischer Moosrasen. Svensk Botanisk Tidskrift, Bd 42, H.4. Pp. 405-443. 
Landucci, M. \& Ludwig T.A.V. 2005. Diatomáceas de rios da bacia hidrográfica Litorânea, PR, Brasil: Coscinodiscophyceae e Fragilariophyceae. Acta Botanica Brasilica 19: 345-357.

Lange-Bertalot, H. 1996. Rote Liste der limnischen Kieselalgen Deutshlands. Schriftenreihe für Vegetationskunde 28: 633-677.

Lange-Bertalot, H. \& Metzeltin, D. 1996. Indicators of Oligotrophy. Iconographia Diatomologica, vol.2. Stuttgart, Koeltz Scientific Books.

Lange-Bertalot, H \& Moser, G. 1994. Brachysira Monographie der Gattung. Bibliotheca Diatomologica, vol. 29. Stuttgart, J. Cramer.

Lobo, E.A.; Callegaro, V.L.M. \& Bender, P. 2002. Utilização de algas diatomáceas epilíticas como indicadoras da qualidade da água em rios e arroios da Região Hidrográfica do Guaíba, RS, Brasil. Santa Cruz do Sul, EDUNISC.

Lobo, E.A.; Callegaro, V.L.M.; Hermany, G.; Bes, D.; Wetzel, C.E. \& Oliveira, M.A. 2004b. Use of epilithic diatoms as bioindicators from lotic systems in southern Brazil, with special emphasis on eutrophication. Acta Limnologica Brasiliensia 16: 25-40.

Lobo, E.A.; Callegaro V.L.M.; Oliveira, M.A.; Salomoni, S.E.; Schuler, S. \& Asai K. 1996. Pollution tolerant diatoms from lotic systems in the Jacui Basin, Rio Grande do Sul, Brazil. Iheringia, Série Botânica 27: 45-72.

Lobo, E.A.; Callegaro, V.L.M.; Wetzel, C.E.; Hermany, G. \& Bes, D. 2004a. Water quality study of the Condor and Capivara Streams, Porto Alegre Municipal District, RS, Brazil, using epilithic diatom biocenoses as bioindicators. Oceanological and Hydrobiological Studies, International Journal of Oceanography and Hydrobiology 33: 77-93.

Mann, D.G.; McDonald, S.M.; Bayer, M.M.; Droop, S.J.M.; Chepurnov, V.A.; Loke, R.E.; Ciobanu, A \& du Buf, J.M.H. 2004. The Sellaphora pupula species complex (Bacillariophyceae): morphometric analysis, ultrastructure and mating data provide evidence for fivr new species. Phycologia 43: 459-482.

Mann, D.G.; Thomas, S.J. \& Evans K.M. 2008. Revision of the diatom genus Sellaphora: a first account of the larger species in the British Isles. Fottea 8: 15-78.

Metzeltin, D. \& Lange-Bertalot, H. 1998. Tropical diatoms of the South America I. Iconographia Diatomologica, vol. 5. Königstein, Koeltz Sicentific Books.

Metzeltin, D. \& Lange-Bertalot, H. 2007. Tropical diatoms of the South America II. Icononographia Diatomologica. vol. 18. Ruggell, A.R.G. Gartner Verlag K.G.

Moser, G. 1999. Die Diatomeenflora von Neukaledonien. Bibliotheca Diatomologica, vol. 43. Stuttgart, Gebr. Borntraeger Verlagsbuchhandlung, Science Publishers.

Paranhos, R. 1996. Alguns métodos para a análise da água. Cadernos Didáticos UFRJ v. 19. SR-1. Rio de Janeiro, UFRJ

Patrick, R. 1940. Diatoms of Northeastern Brazil. Part I - Coscinodiscaceae, Fragilariaceae and Eunotiaceae. Proceedings of the Academy of Natural Sciences of Philadelphia 92: 191-226.

Patrick, R. \& Reimer, C.W. 1966. The diatoms of the United States. vol. 1. Philadelphia, Academy of Natural Sciences of Philadelphia.

Petersen, J.B. 1950. Observations on some small species of Eunotia. Dansk Botanisk Arkiv 14: 1-19.
Procopiak, L.K.; Fernandes, L.F. \& Moreira Filho, H., 2006. Diatomáceas (Bacillariophyta) marinhas e estuarinas do Paraná, Sul do Brasil: lista de espécies com ênfase em espécies nocivas. Biota Neotropica 6: 1-28.

http://www.biotaneotropica.org.br/v6n3/pt/abstract?inventory+ bn02306032006. Acesso em 16/07/2009).

Round, F.E.; Crawford, R.M. \& Mann, D.G. 1990. The diatoms: biology and morphology of genera. Cambridge, Cambridge University Press.

Round, F.E. \& Mann, D.G. 1981. The diatom genus Brachysira. I. Typification and separation from Anomoeoneis. Archiv fur Protistenkunde 124: 221-231.

Sala, S.E.; Duque S.R.; Núñez-Avellaneda M. \& Lamaro A.A. 2002. Diatoms from the colombian Amazon: some species of the genus Eunotia (Bacillariophyceae). Acta Amazonica 32: 589-603.

Salomoni, S.E.; Rocha, O.; Callegaro, V.L. \& Lobo, E.A. 2006. Epilithic diatoms as indicators of water quality in the Gravataí river, Rio Grande do Sul, Brazil. Hydrobiologia 559: 233-246.

Salomoni, S.E. \& Torgan, L.C. 2008. Epilithic diatoms as organic contamination degree indicators in Guaíba Lake, Southern Brazil. Acta Limnologica Brasiliensia 20: 313-324.

Schneck, F.; Torgan, L.C \& Schwarzbold, A. 2007. Epilithic diatom community in high altitude stream impacted by fishfarming in southern Brazil. Acta Limnologica Brasiliensia 19: 341-355.

Schneck, F.; Torgan, L.C \& Schwarzbold, A. 2008. Diatomáceas epilíticas em riacho de altitude no Sul do Brasil. Rodriguésia 59: 325-338.

Soares, M.C.S.; Sophia, M.G. \& Huszar, V.L.M. 2007. Phytoplankton flora of two rivers in Southeast Brazil. Revista Brasileira de Botânica 30: $433-450$.

Stevenson, R.J. 1996. An introduction to algal ecology in freshwater benthic habitats In Stevenson, R.J., Bothwell, M.L. \& Lowe, R.L. (Eds.). Algal Ecology. Freshwater Benthic Ecosystems. San Diego, Academic Press.

Tomas, C.R. (Ed.). 1996. Identifying Marine Diatoms and Dinoflagellates. San Diego, Academic Press.

Torgan, L.C.; Paula, M.C.F. \& Delani, O.M. 1993. Diatomáceas (Bacillariophyceae) perifíticas em Sphagnum recurvum P. Beauv., no Parque Estadual de Itapuã, Rio Grande do Sul, Brasil: taxonomia e aspectos ecológicos. Iheringia, Série Botânica 5: 109-142.

Tremarin, P.I.; Ludwig, T.A.V. \& Moreira-Filho, H., 2008. Eunotia Ehrenberg (Bacillariophyceae) do rio Guaraguaçu, litoral do Paraná, Brasil. Acta Botanica Brasilica 22: 845-862.

Vyverman, W. \& Compère, P. 1991. Nupela giluwensis gen. \& spec. nov. A new genus of naviculoid diatoms. Diatom Research 6: 175-179.

Wetzel, C.E.; Lobo, E.A.; Oliveira, M.A.; Bes, D. \& Hermany, G. 2002. Diatomáceas epilíticas relacionadas a fatores ambientais em diferentes trechos dos Rios Pardo e Pardinho, bacia hidrográfica do Rio Pardo, RS, Brasil: resultados preliminares. Caderno de Pesquisa Ser. Bio. 14: $17-38$

Williams, D.M. 1990. Fragilaria floridana Hanna: ultraestructure of the valve and girdle and its transference to Fragilariforma Williams \& Round. In: Pp. 259-265. Ricard, M. (Ed.). Ouvrage dédié à la Mémorie du Professeur Henry Germain (1903-1989). Koenigstein, Koeltz 